\section{Cardiovascular events prior to or early after diagnosis of systemic lupus erythematosus in the systemic lupus international collaborating clinics cohort}

M B Urowitz, ${ }^{1}$ D D Gladman, ${ }^{1}$ N M Anderson, ${ }^{1} \mathrm{~J} \mathrm{Su},{ }^{1} \mathrm{~J}$ Romero-Diaz, ${ }^{2}$ S C Bae, ${ }^{3}$ P R Fortin, ${ }^{4} \mathrm{~J}$ Sanchez-Guerrero, ${ }^{1}$ A Clarke, ${ }^{5} \mathrm{~S}$ Bernatsky, ${ }^{6} \mathrm{C}$ Gordon, ${ }^{7} \mathrm{~J}$ G Hanly, ${ }^{8}$ D J Wallace, ${ }^{9} \mathrm{D}$ Isenberg, ${ }^{10}$ A Rahman, ${ }^{10} \mathrm{~J}$ Merrill, ${ }^{11} \mathrm{E}$ Ginzler, ${ }^{12} \mathrm{G}$ S Alarcón, ${ }^{13}$ B F Fessler, ${ }^{13} \mathrm{M}$ Petri, ${ }^{14}$ I N Bruce, ${ }^{15,16} \mathrm{M}$ Khamashta, ${ }^{17} \mathrm{C}$ Aranow, ${ }^{18} \mathrm{M}$ Dooley, ${ }^{19}$ S Manzi, ${ }^{20} \mathrm{R}$ Ramsey-Goldman, ${ }^{21} \mathrm{G}$ Sturfelt, ${ }^{22} \mathrm{O}$ Nived, ${ }^{22} \mathrm{~K}$ Steinsson, ${ }^{23}$ A Zoma, ${ }^{24} \mathrm{G}$ Ruiz-Irastorza, ${ }^{25} \mathrm{~S} \mathrm{Lim},{ }^{26} \mathrm{~K} \mathrm{C}$ Kalunian, ${ }^{27} \mathrm{M}$ Inanç, ${ }^{28}$ $\mathrm{R}$ van Vollenhoven, ${ }^{29} \mathrm{M}$ Ramos-Casals, ${ }^{30} \mathrm{D}$ L Kamen, ${ }^{31} \mathrm{~S}$ Jacobsen, ${ }^{32}$ C Peschken, ${ }^{33}$ A Askanase, ${ }^{34} \mathrm{~T}^{\text {Stoll }}{ }^{35}$

To cite: Urowitz MB, Gladman DD, Anderson NM, et al. Cardiovascular events prior to or early after diagnosis of systemic lupus erythematosus in the systemic lupus international collaborating clinics cohort. Lupus Science \& Medicine 2016;3:e000143.

doi:10.1136/lupus-2015000143

Received 18 December 2015 Revised 18 March 2016 Accepted 23 March 2016

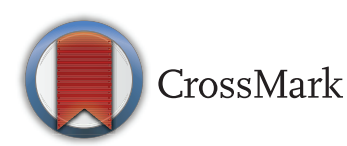

For numbered affiliations see end of article.

Correspondence to Dr Murray B Urowitz; m.urowitz@utoronto.ca

\section{ABSTRACT}

Objective: To describe the frequency of myocardial infarction (MI) prior to the diagnosis of systemic lupus erythematosus (SLE) and within the first 2 years of follow-up.

Methods: The systemic lupus international collaborating clinics (SLICC) atherosclerosis inception cohort enters patients within 15 months of SLE diagnosis. Mls were reported and attributed on a specialised vascular event form. Mls were confirmed by one or more of the following: abnormal ECG, typical or atypical symptoms with ECG abnormalities and elevated enzymes ( $\geq 2$ times upper limit of normal), or abnormal stress test, echocardiogram, nuclear scan or angiogram. Descriptive statistics were used.

Results: 31 of 1848 patients who entered the cohort had an MI. Of those, 23 patients had an MI prior to SLE diagnosis or within the first 2 years of disease. Of the 23 patients studied, $60.9 \%$ were female, $78.3 \%$ were Caucasian, $8.7 \%$ black, $8.7 \%$ Hispanic and $4.3 \%$ other. The mean age at SLE diagnosis was 52.5 \pm 15.0 years. Of the $23 \mathrm{Mls}$ that occurred, $16 \mathrm{Mls}$ occurred at a mean of $6.1 \pm 7.0$ years prior to diagnosis and 7 occurred within the first 2 years of follow-up. Risk factors associated with early MI in univariate analysis are male sex, Caucasian, older age at diagnosis, hypertension, hypercholesterolaemia, family history of $\mathrm{MI}$ and smoking. In multivariate analysis only age $(\mathrm{OR}=1.0695 \% \mathrm{Cl} 1.03$ to 1.09$)$, hypertension (OR=5.01, 95\% $\mathrm{Cl} 1.38$ to 18.23), hypercholesterolaemia $(\mathrm{OR}=4.43,95 \% \mathrm{Cl} 1.51$ to 12.99) and smoking (OR=7.50, 95\% $\mathrm{Cl} 2.38$ to 23.57$)$ remained significant risk factors.

Conclusions: In some patients with lupus, MI may develop even before the diagnosis of SLE or shortly thereafter, suggesting that there may be a link between autoimmune inflammation and atherosclerosis.

\section{INTRODUCTION}

Survival in systemic lupus erythematosus (SLE) has improved significantly over the past four decades. ${ }^{12}$ Nevertheless, the mortality risk in SLE remains three times that of the general population. The major causes of death remain active lupus, infection and cardiovascular disease. ${ }^{2}{ }^{3}$ It has long been known that mortality patterns in SLE have suggested that early deaths are due to lupus and infection, whereas late deaths are often associated with cardiovascular disease. ${ }^{4}$ Indeed, cardiovascular disease has been recognised as a major morbidity among patients with SLE. ${ }^{15-7}$ The prevailing concept has been that atherosclerosis develops prematurely among patients with SLE in the setting of chronic inflammatory disease, with a contribution from increased traditional cardiovascular risk factors. Similar observations have been made in a number of inflammatory musculoskeletal diseases such as rheumatoid arthritis ${ }^{8}$ and psoriatic arthritis. $^{9} 10$ Recently, Pahau et $a l^{11}$ reported increased prevalence of cardiovascular disease prior to the onset of rheumatoid arthritis. Bartels et $a l^{12}$ observed an excess of cardiovascular events in the 2 years prior to the diagnosis of SLE in an analysis of a US 
population-based study and suggested that this may be due to either delayed SLE diagnosis or actual accelerated cardiovascular disease prior to SLE.

The systemic lupus international collaborating clinics (SLICC) group has developed an international registry of newly diagnosed (within 15 months of diagnosis) patients with SLE to carry out a prospective, longitudinal study to determine the incidence, prevalence and nature of atherosclerotic coronary artery disease (CAD) in SLE, to identify associated risk factors for the development of CAD and its outcomes and to discern the contribution of disease and therapy to the occurrences of these risk factors. ${ }^{13-15}$ In the course of observation of this SLICC cohort, atherosclerotic cardiovascular events were identified either prior to the diagnosis of SLE or soon thereafter. The aim of this study was to examine the frequency of myocardial infarction (MI) prior to the diagnosis of SLE and within the first 2 years of follow-up.

\section{METHODS}

The SLICC group includes investigators interested in the study of outcomes in SLE and comprises 33 centres from 11 countries, in North America, Europe and Asia. ${ }^{13-15}$ An inception cohort of patients with SLE has been assembled and followed at yearly intervals according to a standardised protocol between 2000 and 2014 . Sample size calculation at the initiation of the cohort suggested that 1800 patients would suffice to identify CAD events even allowing for loss to follow-up. Patient accrual occurred from 2000 to 2011.

\section{Patient selection}

Patients are eligible to enter the cohort within 15 months of SLE diagnosis, which is determined when a patient has achieved four or more American College of Rheumatology (ACR) criteria for the classification of SLE. ${ }^{16}$ All patients consented to participate in the study which was approved by the local institutional ethic boards.

\section{Assessments}

Clinical and laboratory features of SLE and co-morbidities, including CAD risk factors, are recorded in a standardised protocol at yearly intervals. Hypertension is defined as elevated blood pressure $>140 / 90 \mathrm{~mm} \mathrm{Hg}$ or receiving antihypertensive therapy. Hypercholesterolaemia is defined as total cholesterol $>5.2 \mathrm{mmol} / \mathrm{L}$ or receiving hyperlipidaemia therapy. Vascular events including MI are described and attributed on a specialised form. ${ }^{14} 15$

\section{Event diagnosis}

MI diagnosis was based on the MONICA (MONItoring of Trends and Determinants in CArdiovascular Disease) criteria defined as by '(a) definite ECG or (b) Symptoms typical or atypical or inadequately described, together with probable ECG and abnormal enzymes, or (c) Symptoms typical and abnormal enzymes with ischemic or noncodable ECG'. ${ }^{17}$ The diagnosis was often supported by abnormal stress test, abnormal echocardiogram, abnormal nuclear scan and abnormal angiogram as reported by the treating institution. We also looked at the occurrence of angina defined by the MONICA criteria as severe pain or discomfort over the upper or lower sternum or anterior left chest and left arm and diagnosed by the clinician as angina and attributed to atherosclerosis (not associated with active lupus). ${ }^{17}$ Since the SLICC cohort began in 2000, these criteria were adopted prior to the universal definition of MI criteria, ${ }^{18}$ but were based on the MONICA criteria. ${ }^{17}$

\section{Ascertainment of previous events}

Events were self-reported and validated from medical records using the criteria listed above.

\section{Statistical analysis}

Descriptive statistics and multivariate logistic regression analysis were used to compare the characteristics of patients with and without MI, using SAS V.9.3. Variables that reached statistical significance in the univariate analysis were included in the regression model. The prevalence of MI in the SLICC cohort between the ages 40 and 49 was compared with the prevalence of selfreported MI in the province of Ontario.

\section{RESULTS}

Between 2000 and 2014, 1848 patients were entered into the cohort with a mean follow-up of $8.9 \pm 3.3$ years. There were 1640 females $(88.8 \%)$, with a mean age at diagnosis of $34.7 \pm 13.3$ years and mean disease duration at entry to the cohort of $5.6 \pm 4.2$ months.

Thirty-one of 1848 patients had an MI. Of those, 23 patients had an MI either prior to SLE diagnosis or within the first 2 years of disease. Of the 23 patients studied, 14 were female, 18 were Caucasian, 2 African descendants, 2 Hispanic and 1 other. The mean age at SLE diagnosis was $52.5 \pm 15.0$ years, and the mean age at MI was $49.3 \pm 13.4$ years (table 1 ). Of the 23 MIs, 16 occurred at a mean of $6.1 \pm 7.0$ years prior to diagnosis and 7 occurred within the first 2 years of follow-up (figure 1). Of the six patients with MI five or more years prior to the SLE diagnosis, three were female, five were Caucasian, the mean age at MI was $49.1( \pm 9)$ years and the mean age at SLE diagnosis was $64.5( \pm 9.2)$ years. There were no demographic differences between patients who had an MI before (16) and after (7) SLE diagnosis.

The demographic and disease characteristics of the 23 patients with an early MI were compared with those who did not have an MI within the SLICC inception cohort (table 1). Coronary artery risk factors found more frequently in those with an early MI were male sex, Caucasian ethnicity, older age at diagnosis, family history of all MIs (not necessarily premature), hypertension, hypercholesterolaemia, smoking and lower SLEDAI-2K at inception. There was no difference in organ 
Table 1 Cohort characteristics and coronary artery disease risk factors at baseline

\begin{tabular}{|c|c|c|c|}
\hline & $\begin{array}{l}\text { MI before or shortly after SLE } \\
\text { Patients }(n=23)\end{array}$ & $\begin{array}{l}\text { No MI } \\
\text { Patients }(n=1825)\end{array}$ & p Value \\
\hline $\operatorname{Sex}(F)$ & $14(60.9 \%)$ & $1626(89.1 \%)$ & $<0.001$ \\
\hline Caucasian & $18(78.3 \%)$ & $879(48.5 \%)$ & 0.005 \\
\hline Mean age at SLE diagnosis & $52.5 \pm 15.0$ & $34.5 \pm 13.2$ & $<0.001$ \\
\hline Mean age at MI diagnosis & $49.3 \pm 13.4$ & NA & NA \\
\hline SLE duration in months & $5.6 \pm 4.2$ & $6.1 \pm 4.5$ & 0.547 \\
\hline SLEDAI-2K & $3.7 \pm 3.5$ & $5.4 \pm 5.4$ & 0.03 \\
\hline Hypercholesterolaemia & $17(73.9 \%)$ & $652(35.7 \%)$ & $<0.001$ \\
\hline Hypertension & $19(82.6 \%)$ & 705 (38.6\%) & $<0.0001$ \\
\hline BP diastolic & $74.2 \pm 12.2$ & $75.2 \pm 11.0$ & 0.67 \\
\hline BP systolic & $125.8 \pm 18.5$ & $119.6 \pm 16.8$ & 0.07 \\
\hline Diabetes & $1(4.6 \%)$ & $68(3.7 \%)$ & 0.34 \\
\hline Metabolic syndrome & $5 / 19(26.3 \%)$ & 238/1638 (13.0\%) & 0.18 \\
\hline Family history of $\mathrm{MI}^{\star}$ & $9 / 23(39.1 \%)$ & $264 / 1752(55.3 \%)$ & 0.008 \\
\hline Smoking ever & $18 / 23(78.3 \%)$ & $629 / 1185(34.6 \%)$ & 0.001 \\
\hline Anti-dsDNA antibodies & $7 / 22(31.8 \%)$ & $650 / 1660(39.1 \%)$ & 0.57 \\
\hline Low complement components 3 and 4 & $8 / 22(36.4 \%)$ & $617 / 1662(37.1 \%)$ & 0.76 \\
\hline High sensitivity $C$ reactive protein & $7 / 16(43.8 \%)$ & $298 / 1263(23.5 \%)$ & 0.06 \\
\hline Erythrocyte sedimentation rate & $8 / 14(57.1 \%)$ & $519 / 928(55.9 \%)$ & 0.91 \\
\hline Anticardiolipin antibodies & $1.47 \pm 6.06$ & $10.42 \pm 83.23$ & 0.66 \\
\hline Lupus anticoagulant & $3 / 17(17.7 \%)$ & $249 / 1190(20.9 \%)$ & 1.00 \\
\hline Corticosteroids & $19(82.6 \%)$ & $1260(69.0 \%)$ & 0.16 \\
\hline Antimalarials & $14(60.9 \%)$ & $1233(67.7 \%)$ & 0.75 \\
\hline Immunosuppressives & $10(43.5 \%)$ & $723(40.0 \%)$ & 0.91 \\
\hline
\end{tabular}

involvement between the two groups. In addition, there was no difference in serological manifestations including positive anti-DNA, low complement or the presence of anticardiolipin antibodies or the lupus anticoagulant at inception. High sensitivity $\mathrm{C}$ reactive protein and erythrocyte sedimentation rate were similar between the two groups at inception. Use of corticosteroids, antimalarials and immunosuppressive medications was similar between the two groups at inception (table 1).

In a logistic regression analysis, risk factors associated with early $\mathrm{MI}$ in univariate analysis were male sex $(\mathrm{OR}=5.25,95 \%$ CI 2.25 to 12.29$)$, Caucasian race
( $\mathrm{OR}=3.77$, 95\% CI 1.39 to 10.19$)$, age $(\mathrm{OR}=1.08,95 \%$ CI 1.05 to 1.11$)$, family history of MI (OR=2.02, 95\% CI 1.03 to 3.99 ), hypertension ( $\mathrm{OR}=7.55,95 \%$ CI 2.56 to 22.27 ), hypercholesterolaemia ( $\mathrm{OR}=5.91,95 \%$ CI 2.17 to 16.10$)$ and smoking ever (OR=6.85, 95\% CI 2.53 to 18.52). Multivariate analysis revealed that only age $(\mathrm{OR}=1.0695 \%$ CI 1.03 to 1.09 ), hypertension (OR=5.09, $95 \%$ CI 1.34 to 18.23), hypercholesterolaemia $(\mathrm{OR}=4.43,95 \%$ CI 1.51 to $12.99)$ and smoking (OR=7.50, 95\% CI 2.38 to 23.57) were independent risk factors for the development of early MI after controlling SLEDAI-2K, low complement and corticosteroids in the model (table 2).
Figure 123 myocardial infarction (MI) events occurred prior to or in the first 2 years in 1848 patients. Age at Ml is indicated by the number below each bar.

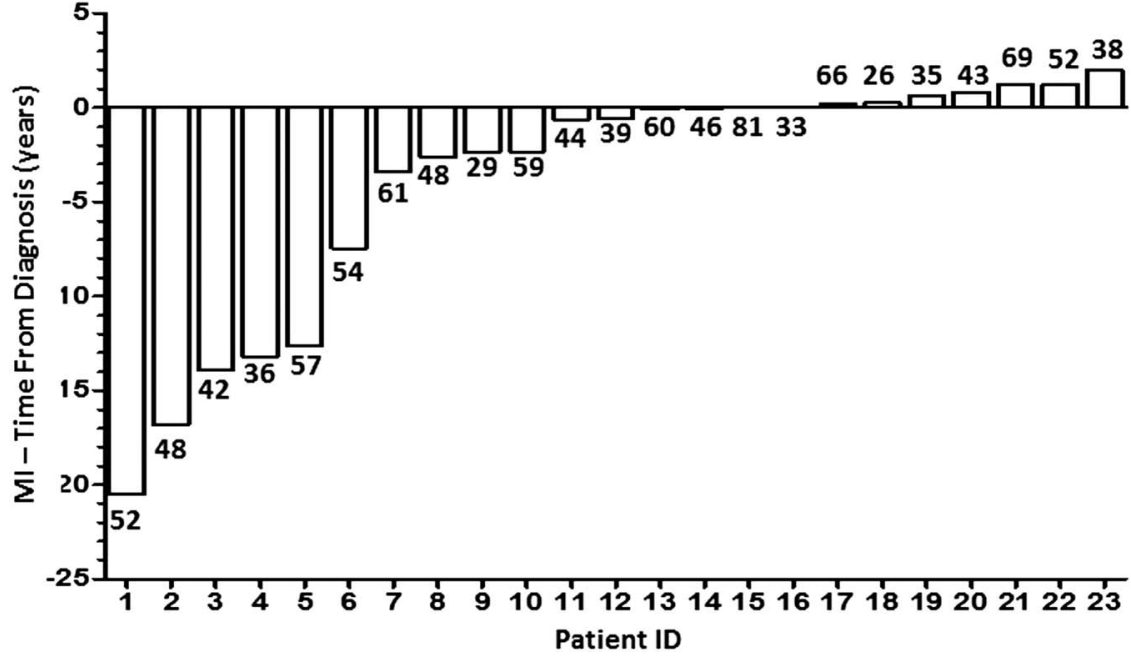


Table 2 Multivariable logistic regression for the outcome of early myocardial infarction

\begin{tabular}{|c|c|c|c|c|}
\hline Predictors & OR & $\begin{array}{l}\text { Lower } \\
95 \% \\
\text { Cl for } \\
\text { OR }\end{array}$ & $\begin{array}{l}\text { Upper } \\
95 \% \\
\text { CI for } \\
\text { OR }\end{array}$ & p Value \\
\hline $\begin{array}{l}\text { Age at systemic lupus } \\
\text { erythematosus diagnosis }\end{array}$ & 1.06 & 1.03 & 1.09 & $<0.0006$ \\
\hline SLEDAI-2K & 0.87 & 0.76 & 1.00 & 0.057 \\
\hline Hypertension & 5.09 & 1.38 & 18.23 & 0.0145 \\
\hline Low complement & 2.34 & 0.78 & 7.04 & 0.131 \\
\hline Corticosteroids & 2.42 & 0.76 & 7.69 & 0.135 \\
\hline Hypercholesterolaemia & 4.43 & 1.51 & 12.99 & 0.0068 \\
\hline Smoking ever & 7.50 & 2.38 & 23.57 & 0.0006 \\
\hline
\end{tabular}

There were 17 patients with a history of MI within 5 years prior to diagnosis or within the first 2 years after diagnosis of SLE whose mean age was 48 years at MI. Therefore, the prevalence of MI in the SLICC cohort between the ages 40 and $49(17 / 325)$ was $4.8 \%$ compared with the province of Ontario in Canada in a population of 5077402 at ages $40-49$ of $0.7 \% .^{19}$

We then examined the frequency of angina due to atherosclerosis. Fifteen patients were diagnosed with angina (without MI) and of these five developed the angina within the first 2 years of SLE at a mean age of $51.0 \pm 17.6$ years.

\section{DISCUSSION}

Atherosclerotic disease is an important cause of morbidity and mortality among patients with SLE. Previous studies have concentrated on cardiovascular events occurring after the diagnosis of SLE. The prevalence of symptomatic CAD in established lupus cohorts varies from $8.3 \%$ in Baltimore, $6.6 \%$ in Pittsburgh and $8.9 \%$ in Toronto. ${ }^{156}$ The mean age at first CAD event is similar in the three cohorts at $48-49$ years. In a communitybased cohort using the California Discharge Database, Ward showed that women with SLE between 18 and 44 years of age were more likely than age-matched controls to be admitted to hospital with MI. ${ }^{20}$ Incidence rates of $\mathrm{CAD}$ in women with lupus are significantly raised compared with age-matched controls. Women with lupus aged 35-44 were 50 times more likely to have an MI compared with age-matched women. ${ }^{5}$ The rate of MI in the Toronto lupus cohort was five times higher than the general population. Furthermore, the mean age of MI in the Toronto lupus cohort was 49 years compared with the peak years of the general population of $65-74$ years. ${ }^{21}$ In addition, Esdaile has shown that traditional Framingham risk factors fail to fully account for the accelerated atherosclerosis in patients with SLE. ${ }^{22}$

These studies indicate that women with SLE have a significantly increased risk for $\mathrm{MI}$, the onset of the CAD is premature and that lupus itself is an important associated factor. In a study of the natural history of hypercholesterolaemia in an inception cohort (within 12 months of diagnosis) of patients with SLE, Bruce et $a l^{23}$ reported that time to event in patients who had a CAD-related event was 7.5 years from diagnosis of SLE.

Until recently, there has been little attention to cardiac events occurring either prior to SLE or early in the course of the disease. In a large US study of mortality and cardiovascular burden in SLE, Bartels et $a l^{12}$ found that prior cardiovascular disease events in the 2 years preceding diagnosis of SLE occurred in $23 \%$ of 70 patients compared with $10 \%$ of 2565 aged and sexmatched controls $(\mathrm{p}<0.05)$.

The SLICC atherosclerosis registry, which is an inception cohort within 15 months of diagnosis, allows identification of atherosclerotic events early in the course of the disease. The current study demonstrates that clinical cardiovascular events may occur prior to or early after the diagnosis of lupus. Of the 1848 patients followed in the SLICC cohort, there were 23 MIs that occurred prior to or within the first 2 years of disease. The prevalence of this occurrence in the SLICC cohort patients aged 40-49 was higher than the self-reported prevalence of MI in Canadian Community Health Survey conducted in Ontario of similar age (4.8\% vs $0.7 \%$, respectively) ${ }^{19}$ Self-reported incidence of MI tends to be over-reported as compared with the prevalence from administrative data and therefore makes the significance of MI prevalence in the SLICC cohort greater. ${ }^{24}$ In addition, five instances of angina without MI were documented within the same time frame and are at risk for developing MI later in their course.
Figure 2 Evolution of benign autoimmunity and early atherosclerosis to clinical disease. SLE, systemic lupus erythematosus.

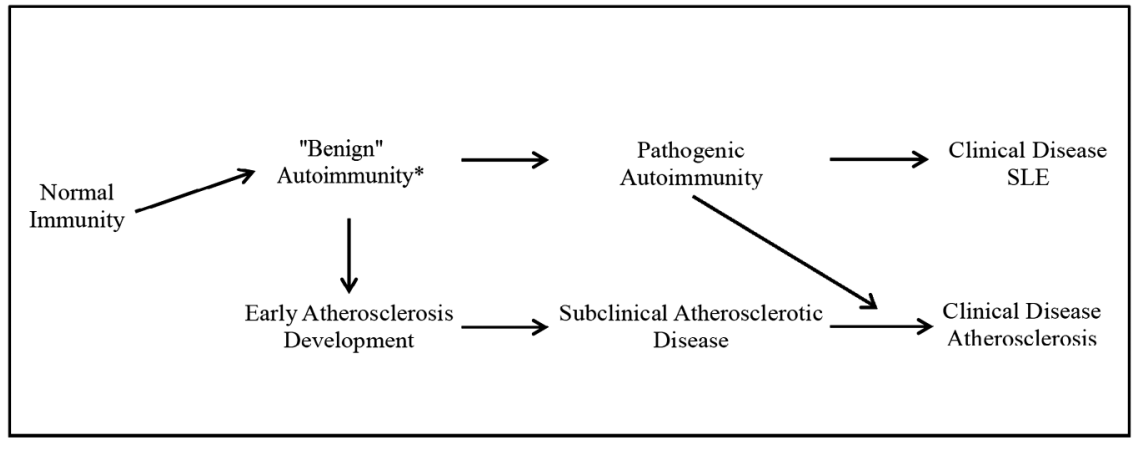


Our study indicates that older males diagnosed with lupus are most at risk for developing MIs early in the course of their lupus. It is interesting that anticardiolipin antibodies, lupus anticoagulant and family history of all MIs at inception were not significantly associated factors in the multivariate analysis.

A limitation of our study is that the universal definition of MI criteria was not available at the time our cohort was developed. However, we did use the WHO criteria that were available at the time. Moreover, since the cohort was created to discern atherosclerotic cardiovascular events in patients with lupus, the investigators excluded the possibility of lupus disease activity (eg, pericarditis, myocarditis, vasculitis and pleurisy) when attributing an event to atherosclerosis.

These findings raise the question as to the relationship between atherosclerotic events and SLE. For atherosclerosis and SLE, the clinical event is obviously preceded by pathogenic mechanisms over a few years. It has been shown that autoantibodies develop in patients with SLE many years before the clinical recognition of the disease. ${ }^{25}$ This could lead to subclinical autoimmunity which subsequently manifests as a clinical diagnosis of SLE (figure 2). Similarly, inflammatory and metabolic mechanisms develop in patients with atherosclerosis before any evidence for clinical disease (MI, angina). ${ }^{26}$ It is therefore possible that subclinical autoimmunity may result in subclinical atherosclerosis and either can manifest itself clinically as the initial clinical presentation. ${ }^{27}$ Alternatively, the two mechanisms, autoimmunity and atherosclerosis, may develop independently but concurrently and may manifest themselves clinically at different times. This may be due to either a common genetic predisposition to both conditions or independent genetic factors interacting through epigenetic or environmental influences.

Our study highlights the possible relationship between immunologic and inflammatory mechanisms leading to both SLE and atherosclerosis. Thus, the physician caring for patients with SLE must be aware that these patients require careful investigation and treatment of cardiovascular risk factors. Also, physicians caring for patients with premature atherosclerotic disease should consider the possibility of an increased risk of autoimmune disease and consider investigating such patients for 'benign autoimmunity' that is the presence of autoantibodies in the absence of any clinical disease with an autoantibody screen.

\footnotetext{
Author affiliations

${ }^{1}$ Centre for Prognosis Studies in the Rheumatic Diseases, Toronto Western Hospital and University of Toronto, Toronto Ontario, Canada

${ }^{2}$ Instituto Nacional de Ciencias Medicas y Nutrición, Mexico City, Mexico

${ }^{3}$ Department of Rheumatology, Hanyang University Hospital for Rheumatic Diseases, Seoul, Korea

${ }^{4}$ Division of Rheumatology, Centre Hospitalier Universitaire de Québec et Université Laval, Quebec City, Canada

${ }^{5}$ Division of Rheumatology, Cumming School of Medicine University of Calgary, Calgary, Alberta, Canada
}

${ }^{6}$ Divisions of Clinical Immunology/Allergy and Clinical Epidemiology, Montreal General Hospital, McGill University Health Centre, Montreal, Quebec, Canada

${ }^{7}$ Rheumatology Research Group, School of Immunity and Infection, College of Medical and Dental Sciences, University of Birmingham, Birmingham, UK

${ }^{8}$ Division of Rheumatology, Department of Medicine and Department of Pathology, Queen Elizabeth II Health Sciences Centre and Dalhousie University, Halifax, Nova Scotia, Canada

${ }^{9}$ Cedars-Sinai/David Geffen School of Medicine at UCLA, Los Angeles,

California, USA

${ }^{10}$ Centre for Rheumatology Research, University College, London, UK

${ }^{11}$ Department of Clinical Pharmacology, Oklahoma Medical Research

Foundation, Oklahoma City, Oklahoma, USA

${ }^{12}$ Department of Medicine, SUNY Downstate Medical Center, Brooklyn, New York, USA

${ }^{13}$ Department of Medicine, Division of Clinical Immunology and Rheumatology, University of Alabama at Birmingham, Birmingham, Alabama, USA

${ }^{14}$ Department of Rheumatology, Johns Hopkins University School of Medicine, Baltimore, Maryland, USA

${ }^{15}$ Arthritis Research UK Centre for Epidemiology, Centre for Musculoskeletal Research, Institute of Inflammation and Repair, Manchester Academic Health Sciences Centre, The University of Manchester, Manchester, UK

${ }^{16}$ NIHR Manchester Musculoskeletal Biomedical Research Unit, Central

Manchester University Hospitals NHS Foundation Trust, Manchester Academic Health Science Centre, London, UK

${ }^{17}$ Lupus Research Unit, The Rayne Institute, St Thomas' Hospital, King's

College London School of Medicine, London, UK

${ }^{18}$ Feinstein Institute for Medical Research, Manhasset, New York, USA

${ }^{19}$ Division of Rheumatology and Immunology, Department of Medicine, University of North Carolina, Chapel Hill, North Carolina, USA

${ }^{20}$ Department of Medicine, West Penn Allegheny, Pittsburgh, Pennsylvania, USA

${ }^{21}$ Northwestern University and Feinberg School of Medicine, Chicago, Illinois, USA

${ }^{22}$ Department of Rheumatology, University Hospital Lund, Lund, Sweden

${ }^{23}$ Department of Rheumatology, Center for Rheumatology Research Fossvogur Landspitali University Hospital, Reyjkavik, Iceland

${ }^{24}$ Lanarkshire Centre for Rheumatology, Hairmyres Hospital, East Kilbride, Scotland, UK

${ }^{25}$ Autoimmune Disease Unit, Department of Internal Medicine, Hospital Universitario Cruces., University of the Basque Country, Barakaldo, Spain

${ }^{26}$ Division of Rheumatology, Emory University School of Medicine, Atlanta, Georgia, USA

${ }^{27}$ UCSD School of Medicine, La Jolla, California, USA

${ }^{28}$ Division of Rheumatology, Department of Internal Medicine, Istanbul Medical Faculty, Istanbul University, Istanbul, Turkey

${ }^{29}$ Unit for Clinical Therapy Research (ClinTRID), The Karolinska Institute, Stockholm, Sweden

${ }^{30}$ Josep Font Autoimmune Diseases Laboratory, IDIBAPS, Department of Autoimmune Diseases, Hospital Clínic, Barcelona, Spain

${ }^{31}$ Division of Rheumatology, Medical University of South Carolina, Charleston, South Carolina, USA

${ }^{32}$ Department of Rheumatology Rigshospitalet, Copenhagen University Hospital, Copenhagen, Denmark

${ }^{33}$ Department of Medicine and Community Health Sciences, University of Manitoba, Winnipeg, Manitoba, Canada

${ }^{34}$ Division of Rheumatology, Columbia University Medical Center, New York, USA

${ }^{35}$ Department of Rheumatology, Kantousspital, Schaffhausen, Switzerland

Acknowledgements The authors gratefully acknowledge the generous donation of our patients' time and the dedication of all the fellows, research coordinators and assistants in the SLICC group to the completion of this work.

Contributors All authors were involved in the study conception and design, acquisition of data, and analysis and interpretation of data. All authors have contributed to this work to a significant extent and have read and approved the submitted version. 
Competing interests None declared.

Ethics approval University Health Network Research Ethics Board.

Funding The authors would like to acknowledge the financial support of the Canadian Institutes of Health Research, Lupus UK, Tolfo Family, Lupus Ontario and the Conn Smythe Foundation.

Provenance and peer review Not commissioned; externally peer reviewed.

Data sharing statement All participants in the SLICC group signed a data and sharing agreement.

Open Access This is an Open Access article distributed in accordance with the Creative Commons Attribution Non Commercial (CC BY-NC 4.0) license, which permits others to distribute, remix, adapt, build upon this work noncommercially, and license their derivative works on different terms, provided the original work is properly cited and the use is non-commercial. See: http:// creativecommons.org/licenses/by-nc/4.0/

\section{REFERENCES}

1. Urowitz MB, Gladman DD, Tom BD, et al. Changing patterns in mortality and disease outcome for patients with systemic lupus erythematosus. J Rheumatol 2008;35:2152-8.

2. Yukovich M, Vostretsova K, Chen W, et al. Overall and cause-specific mortality in patients with systemic lupus erythematosus: a meta-analysis of observational studies. Arthritis Care Res (Hoboken) 2014;66:608-16.

3. Abu-Shakra M, Urowitz MB, Gladman DD, et al. Mortality studies in systemic lupus erythematosus. Results from a single centre. I. Causes of death. J Rheumatol 1995;22:1259-64.

4. Urowitz MB, Bookman AA, Koehler BE, et al. The bimodal mortality in systemic lupus erythematosus. Am J Med 1976;60:221-5.

5. Manzi S, Meilahn EN, Rairie JE, et al. Age-specific incidence rates of myocardial infarction and angina in women with systemic lupus erythematosus: comparison with the Framingham Study. Am J Epidemiol 1997; 145:408-5.

6. Petri M, Perez-Gutthann S, Spence D, et al. Risk factors for coronary artery disease in patients with systemic lupus erythematosus. Am J Med 1992;93:513-19.

7. Nikpour M, Urowitz MB, Gladman DD. Epidemiology of atherosclerosis in systemic lupus erythematosus. Curr Rheumatol Rep 2009;11:248-54.

8. Maradit-Kremers H, Nicola PJ, Crowson CS, et al. Cardiovascular death in rheumatoid arthritis: a population-based study. Arthritis Rheum 2005;52:722-32.

9. Gladman DD, Ang M, Su L, et al. Cardiovascular morbidity in psoriatic arthritis (PsA). Ann Rheum Dis 2009;68:1131-5.

10. Gonzalez-Juanatey C, Llorca J, Amigo-Diaz E, et al. High prevalence of subclinical atherosclerosis in psoriatic arthritis patients without clinical evident cardiovascular disease or classic cardiovascular risk factors. Arthritis Rheum 2007;57:1-74-80.

11. Pahau H, Brown MA, Paul S, et al. Cardiovascular disease is increased prior to onset of rheumatoid arthritis but not osteoarthritis: the population-based Nord-Trøndelag health study (HUNT). Arthritis Res Ther 2014;16:R85.

12. Bartels CM, Buhr KA, Goldberg JW, et al. Mortality and cardiovascular burden of systemic lupus erythematosus in a US population-based cohort. J Rheumatol 2014;41:680-7.

13. Urowitz MB, Gladman DD. Atherosclerosis and lupus-the SLICC study. Lupus 2007;16;925-8.

14. Urowitz MB, Gladman D, Ibañez, et al., Systemic Lupus International Collaborating Clinics (SLICC). Clinical manifestations and coronary artery disease risk factors at diagnosis of systemic lupus erythematosus: data from an international inception cohort. Lupus 2007; $16: 731-5$

15. Urowitz MB, Gladman D, Ibañez D, et al., Systemic Lupus International Collaborating Clinics (SLICC). Accumulation of coronary artery disease risk factors over three years: data from an International Inception Cohort. Arthritis Rheum 2008;59: 176-80.

16. Tan EM, Cohen AS, Fries JF, et al. The 1982 revised criteria for the classification of systemic lupus erythematosus. Arthritis Rheum 1982;25:1271-7.

17. Tunstall-Pedoe $\mathrm{H}$, Kuulasmaa $\mathrm{K}$, Amouyel $\mathrm{P}$, et al. Myocardial infarction and coronary deaths in the World Health Organization MONICA Project. Registration procedures, event rates, and case-fatality rates in 38 populations from 21 countries in four continents. Circulation 1994;90:583-612.

18. Thygesen K, Alpert JS, White HD, et al. Universal definition of myocardial infarction. Circulation 2007;116:2634-53.

19. Chow C, Donovan L, Manuel D, et al. for the Canadian Cardiovascular Outcomes Research Team. Regional variation in self-reported heart disease prevalence in Canada. Can J Cardiol 2005;21:1265-71.

20. Ward MM. Premature morbidity from cardiovascular and cerebrovascular diseases in women with systemic lupus erythematosus. Arthritis Rheum 1999;42:338-46.

21. Nikpour M, Urowitz MB, Gladman DD. Premature atherosclerosis in systemic lupus erythematosus. Rheum Dis Clin North Am 2005;31:329-54.

22. Esdaile JM, Abrahamowicz M, Grodzicky T, et al. Traditional Framingham risk factors fail to fully account for accelerated atherosclerosis in systemic lupus erythematosus. Arthritis Rheum 2001;44:2331-7.

23. Bruce IN, Urowitz MB, Gladman DD, et al. The natural history of hypercholesterolaemia in SLE. J Rheumatol 1999;26: 2137-43.

24. Muggah E, Graves E, Bennett C, et al. Ascertainment of chronic disease using population health data: a comparison of health administrative data and patient self-report. BMC Public Health 2013:13:16.

25. Arbuckle MR, McCain MT, Rubertone MV, et al. Development of autoantibodies before the clinical onset of systemic lupus erythematosus. N Engl J Med 2003;349:1526-33.

26. Galkina E, Ley K. Immune and inflammatory mechanisms of atherosclerosis. Annu Rev Immunol 2009;27:165-97.

27. Majka DS, Chang RW. Is preclinical autoimmunity benign? The case of cardiovascular disease. Rheum Dis Clin North Am 2014;40:659-68. 
Erratum: Cardiovascular events prior to or early after diagnosis of systemic lupus erythematosus in the systemic lupus international collaborating clinics cohort

Urowitz MB, Gladman DD, Anderson NM, et al. Cardiovascular events prior to or early after diagnosis of systemic lupus erythematosus in the systemic lupus international collaborating clinics cohort. Lupus Sci Med 2016;3:e000143. doi:10.1136/ lupus-2015-000143

There is an error in table 1 of this article.

In table 1: Cohort characteristics and coronary artery disease risk factors at baseline.

The entry in the row 'Family history of MI' under the column 'No MI patients' should read 264/1752 (15.1\%).

Lupus Science \& Medicine 2016;3:e000143corr1. doi:10.1136/lupus-2015-000143corr1 